\title{
Density in Spaces of Interpolation by Hankel Translates of a Basis Function
}

\author{
Cristian Arteaga and Isabel Marrero \\ Departamento de Análisis Matemático, Universidad de La Laguna, 38271 La Laguna (Tenerife), Spain
}

Correspondence should be addressed to Isabel Marrero; imarrero@ull.es

Received 3 May 2013; Accepted 24 June 2013

Academic Editor: Józef Banaś

Copyright ( 2013 C. Arteaga and I. Marrero. This is an open access article distributed under the Creative Commons Attribution License, which permits unrestricted use, distribution, and reproduction in any medium, provided the original work is properly cited.

The function spaces $Y_{m}\left(m \in \mathbb{Z}_{+}\right)$arising in the theory of interpolation by Hankel translates of a basis function, as developed by the authors elsewhere, are defined through a seminorm which is expressed in terms of the Hankel transform of each function and involves a weight $w$. At least two special classes of weights allow to write these indirect seminorms in direct form, that is, in terms of the function itself rather than its Hankel transform. In this paper, we give fairly general conditions on $w$ which ensure that the Zemanian spaces $\mathscr{B}_{\mu}$ and $\mathscr{H}_{\mu}(\mu>-1 / 2)$ are dense in $Y_{m}\left(m \in \mathbb{Z}_{+}\right)$. These conditions are shown to be satisfied by the weights giving rise to direct seminorms of the so-called type II.

\section{Introduction}

The Hankel integral transformation is usually defined by

$$
\left(h_{\mu} \varphi\right)(x)=\int_{0}^{\infty} \varphi(t) \mathscr{J}_{\mu}(x t) d t \quad(x \in I)
$$

where $I=] 0, \infty\left[, \mathscr{J}_{\mu}(z)=z^{1 / 2} J_{\mu}(z)(z \in I)\right.$, and $J_{\mu}$ denotes the Bessel function of the first kind and order $\mu \in \mathbb{R}$.

1.1. The Distributional Hankel Transformation. Aiming to define the Hankel transformation in spaces of distributions, Zemanian [1] introduced the space $\mathscr{H}_{\mu}$ of all those smooth, complex-valued functions $\varphi=\varphi(x)(x \in I)$ such that

$$
\begin{array}{r}
v_{\mu, r}(\varphi)=\max _{0 \leq k \leq r} \sup _{x \in I}\left|\left(1+x^{2}\right)^{r}\left(x^{-1} D\right)^{k} x^{-\mu-1 / 2} \varphi(x)\right|<\infty \\
\left(r \in \mathbb{Z}_{+}\right) .
\end{array}
$$

Here, and in the sequel, $D=D_{x}=d / d x$. When topologized by the family of norms $\left\{\nu_{\mu, r}\right\}_{r \in \mathbb{Z}_{+}}, \mathscr{H}_{\mu}$ becomes a Fréchet space where $h_{\mu}$ is an automorphism provided that $\mu \geq-1 / 2$. Then the generalized Hankel transformation $h_{\mu}^{\prime}$, defined by transposition on the dual $\mathscr{H}_{\mu}^{\prime}$ of $\mathscr{H}_{\mu}$, is an automorphism of
$\mathscr{H}_{\mu}^{\prime}$ when this latter space is endowed with either its weak ${ }^{*}$ or its strong topology.

Zemanian [2] also constructed the space $\mathscr{B}_{\mu}$ as follows. For every $a>0, \mathscr{B}_{\mu, a}$ consists of all those $\varphi \in \mathscr{H}_{\mu}$ such that $\varphi(x)=0$ whenever $x>a$. This space is endowed with the topology generated by the family of seminorms $\left\{\gamma_{\mu, r}\right\}_{r \in \mathbb{Z}_{+}}$, given by

$$
\begin{array}{r}
\gamma_{\mu, r}(\varphi)=\sup _{x \in] 0, a[}\left|\left(x^{-1} D\right)^{r} x^{-\mu-1 / 2} \varphi(x)\right| \\
\left(\varphi \in \mathscr{B}_{\mu, a}, r \in \mathbb{Z}_{+}\right) .
\end{array}
$$

In this way $\mathscr{B}_{\mu, a}$ becomes a Fréchet space. Moreover, if $0<$ $a<b$, then $\mathscr{B}_{\mu, a} \subset \mathscr{B}_{\mu, b}$ and the topology of $\mathscr{B}_{\mu, a}$ coincides with that inherited from $\mathscr{B}_{\mu, b}$. This allows to consider the inductive limit $\mathscr{B}_{\mu}=\bigcup_{a>0} \mathscr{B}_{\mu, a}$. As usual, the dual spaces of $\mathscr{B}_{\mu}$ and $\mathscr{B}_{\mu, a}$ are respectively denoted by $\mathscr{B}_{\mu}^{\prime}$ and $\mathscr{B}_{\mu, a}^{\prime}(a>$ $0)$. Since $\mathscr{B}_{\mu}$ is a dense subspace of $\mathscr{H}_{\mu}$, $\mathscr{H}_{\mu}^{\prime}$ can be regarded as a subspace of $\mathscr{B}_{\mu}^{\prime}$.

1.2. The Delsarte Kernel and the Hankel Convolution. Hirschman [3], Cholewinski [4], and Haimo [5] developed a convolution theory on Lebesgue spaces for a variant of the 
Hankel transformation closely connected to (1). For $\mu>$ $-1 / 2$, straightforward manipulations of the results in [3-5] allow us to define a convolution for $h_{\mu}$ as follows. Whenever the integrals involved exist, the Hankel convolution of the functions $\varphi=\varphi(x)$ and $\phi=\phi(x)(x \in I)$ is defined as the function

$$
(\varphi \# \phi)(x)=\int_{0}^{\infty} \varphi(y)\left(\tau_{x} \phi\right)(y) d y \quad(x \in I),
$$

where the Hankel translate $\tau_{x} \phi$ of $\phi$ is given by

$$
\left(\tau_{x} \phi\right)(y)=\int_{0}^{\infty} \phi(z) D_{\mu}(x, y, z) d z \quad(x, y \in I) .
$$

Here

$$
\begin{array}{r}
D_{\mu}(x, y, z)=\int_{0}^{\infty} t^{-\mu-1 / 2} \mathscr{J}_{\mu}(x t) \mathscr{J}_{\mu}(y t) \mathscr{J}_{\mu}(z t) d t \\
(x, y, z \in I)
\end{array}
$$

is the so-called Delsarte kernel. Recall that

$$
\begin{array}{r}
D_{\mu}(x, y, z)=\frac{2^{\mu-1}}{\Gamma(\mu+1 / 2) \pi^{1 / 2}}(x y z)^{1 / 2-\mu} \Delta(x, y, z)^{2 \mu-1} \\
(x, y, z \in I),
\end{array}
$$

$\Delta(x, y, z)$ being the area of the triangle with sides of length $x, y, z$ if such a triangle exists, and zero otherwise [3, p. 308]. We note that $D_{\mu}(x, y, z) \neq 0$ only when $|x-y|<z<x+$ $y(x, y, z \in I)$. Furthermore,

$$
\int_{0}^{\infty} D_{\mu}(x, y, z) z^{\mu+1 / 2} d z=c_{\mu}^{-1}(x y)^{\mu+1 / 2} \quad(x, y \in I),
$$

with $c_{\mu}=2^{\mu} \Gamma(\mu+1)$.

For $1 \leq p<\infty$, denote by $L_{\mu}^{p}$ the space of Lebesgue measurable functions whose $p$ th power is absolutely integrable on $I$ with respect to the weight $t^{\mu+1 / 2}$, normed with

$$
\|u\|_{\mu, p}=\left(\int_{0}^{\infty}|u(t)|^{p} t^{\mu+1 / 2} d t\right)^{1 / p} \quad\left(u \in L_{\mu}^{p}\right) .
$$

By $L_{\mu}^{\infty}$ we will represent the space of Lebesgue measurable functions $u=u(t)(t \in I)$ such that $t^{-\mu-1 / 2} u(t)(t \in I)$ is essentially bounded, normed with

$$
\|u\|_{\mu, \infty}=\operatorname{esssup}_{t \in I}\left|t^{-\mu-1 / 2} u(t)\right| \quad\left(u \in L_{\mu}^{\infty}\right) .
$$

If $\varphi \in L_{\mu}^{1}$ and $\phi \in L_{\mu}^{\infty}$, then (5) and (4) exist as continuous functions on $I$. If $\varphi, \phi \in L_{\mu}^{1}$, then (5) and (4) exist as functions in $L_{\mu}^{1}$, and, moreover, the formula

$$
h_{\mu}\left(\tau_{y} \phi\right)(x)=x^{-\mu-1 / 2} \mathscr{J}_{\mu}(x y)\left(h_{\mu} \phi\right)(x) \quad(x, y \in I)
$$

and the exchange formula

$$
h_{\mu}(\varphi \# \phi)(x)=x^{-\mu-1 / 2}\left(h_{\mu} \varphi\right)(x)\left(h_{\mu} \phi\right)(x) \quad(x \in I)
$$

hold.
The study of the Hankel convolution on compactly supported distributions was initiated by de Sousa Pinto [6], only for $\mu=0$. In a series of papers, Betancor and the second named author investigated systematically the generalized \#convolution in wider spaces of distributions, allowing $\mu>$ $-1 / 2$. In this context (cf. [7]), the Hankel translation was shown to be a continuous operator from $\mathscr{H}_{\mu}$ into itself. Thus, the Hankel convolution $f \# \varphi \in \mathscr{H}_{\mu}^{\prime}$ of $f \in \mathscr{H}_{\mu}^{\prime}$ and $\varphi \in \mathscr{H}_{\mu}$ can be defined through

$$
(f \# \varphi)(x)=\left\langle f, \tau_{x} \varphi\right\rangle \quad(x \in I)
$$

[7, Definition 3.1]. The formulas

$$
\begin{gathered}
h_{\mu}^{\prime}\left(\tau_{y} f\right)(x)=x^{-\mu-1 / 2} \mathscr{F}_{\mu}(x y)\left(h_{\mu}^{\prime} f\right)(x) \quad(y \in I), \\
h_{\mu}^{\prime}(f \# \varphi)(x)=x^{-\mu-1 / 2}\left(h_{\mu} \varphi\right)(x)\left(h_{\mu}^{\prime} f\right)(x),
\end{gathered}
$$

respectively extending (11) and (12), hold in the sense of equality in $\mathscr{H}_{\mu}^{\prime}$ (cf. [7, Proposition 3.5]).

\subsection{Interpolation by Hankel Translates of a Basis Function.} In approximation theory, radially symmetric, (conditionally) positive definite functions are used to solve scattered data interpolation problems in Euclidean space. The setting for a variational approach to such interpolation problems, the socalled native spaces, was constructed by several authors upon seminal work of Micchelli [8] and Madych and Nelson [9-11]. Later, Light and Wayne [12] ideated an alternative approach in which the distributional theory of the Fourier transformation plays a prominent role.

When dealing with interpolation by radial basis functions, one can either (i) keep treating the involved functions as radially symmetric functions on $\mathbb{R}^{d}(d \in \mathbb{N}$ ), or (ii) identify them with functions on the positive real half-axis. For instance, Schaback and Wu [13] devised a general theory which allows to write multivariate Fourier transforms or convolutions of radial functions as very simple univariate operations. Motivated by [12], in [14] we benefited from the Hankel transformation and the Hankel convolution in order to provide (ii) with an adequate theoretical support. This new approach generalizes and improves (i) in a sense that is made precise next.

Recall that if $d \in \mathbb{N}$ and $f(x)=f_{0}(|x|)$ (a.e. $x \in \mathbb{R}^{d}$ ) is an integrable radial function, then its $d$-dimensional Fourier transform

$$
\widehat{f}(\xi)=\int_{\mathbb{R}^{d}} f(x) e^{-2 \pi i x \cdot \xi} d x \quad\left(\xi \in \mathbb{R}^{d}\right)
$$

is also radial and reduces to a 1-dimensional Hankel transform of order $d / 2-1$ [15, Theorem 3.3]:

$$
\begin{aligned}
\widehat{f}(\xi)=F_{0}(|\xi|)= & 2 \pi|\xi|^{-(d / 2-1)} \\
& \times \int_{0}^{\infty} f_{0}(s) J_{d / 2-1}(2 \pi|\xi| s) s^{d / 2} d s
\end{aligned}
$$


Actually, since

$$
J_{-1 / 2}(z)=\left(\frac{2}{\pi z}\right)^{1 / 2} \cos z \quad(z \in I),
$$

it turns out that on radial univariate -even- functions, the Fourier transformation, which agrees with the Fourier-cosine transformation, coincides (up to a multiplicative constant) with the Hankel transform as well. Similarly, the abovementioned variant of the Hankel convolution of order $\mu=$ $-1 / 2$ can be seen to coincide with the usual convolution on $\mathbb{R}$ (cf. [16, Example 3.2]). Thus, for $2 \mu+2 \notin \mathbb{N}$ the Hankel convolution structure provides a strict generalization of the Fourier one.

Denote by $L_{\mu, l}^{1}$ the class of all those Lebesgue measurable functions $u=u(t)(t \in I)$ such that

$$
\int_{0}^{a}|u(t)| t^{\mu+1 / 2} d t<\infty \quad(a>0) .
$$

The following spaces were introduced in [14].

Definition 1. Let $w=w(t)>0(t \in I)$ be a continuous function, let

$$
S_{\mu}=S_{\mu, t}=t^{-\mu-1 / 2} D_{t} t^{2 \mu+1} D_{t} t^{-\mu-1 / 2}
$$

be the Bessel differential operator, and let

$$
Y_{m}=\left\{f \in \mathscr{H}_{\mu}^{\prime}: h_{\mu}^{\prime} S_{\mu}^{m} f \in L_{\mu, l}^{1} \cap L_{\mu, w}^{2}\right\} \quad\left(m \in \mathbb{Z}_{+}\right),
$$

where $S_{\mu}^{0}$ is the identity operator, $S_{\mu}^{m}(m \in \mathbb{N})$ is the operator $S_{\mu}$ iterated $m$ times, and $L_{\mu, w}^{2}$ stands for the class of all measurable functions $u=u(t)(t \in I)$ satisfying

$$
\|u\|_{\mu, w}=\left(\int_{0}^{\infty}|u(t)|^{2} w(t) t^{\mu+1 / 2} d t\right)^{1 / 2}<\infty .
$$

A seminorm (norm if $m=0$ ) is defined on $Y_{m}$ by setting

$$
|f|_{m}=\left(\int_{0}^{\infty}\left|\left(h_{\mu}^{\prime} S_{\mu}^{m} f\right)(t)\right|^{2} w(t) t^{\mu+1 / 2} d t\right)^{1 / 2} \quad\left(f \in Y_{m}\right) .
$$

In [14], for $m \in \mathbb{N}$ and suitable conditions on the weight $w$ related to the values of $m$, the spaces $Y_{m}$ were shown to consist of continuous functions on $I$. Also, interpolants to $f \in Y_{m}$ of the form

$$
(U f)(x)=\sum_{i=1}^{n} \alpha_{i}\left(\tau_{a_{i}} \Phi\right)(x)+\sum_{j=0}^{m-1} \beta_{j} p_{\mu, j}(x) \quad(x \in I)
$$

were obtained, where $\left\{a_{1}, \ldots, a_{n}\right\} \subset I$ is the set of interpolation points; $\Phi \in \mathscr{H}_{\mu}^{\prime}$ is a complex function defined on $I$ (the so-called basis function), connected with $w$ through the distributional identity

$$
t^{4 m}\left(h_{\mu}^{\prime} \Phi\right)(t)=\frac{1}{w(t)}
$$

$p_{\mu, j}(x)=x^{2 j+\mu+1 / 2}\left(j \in \mathbb{Z}_{+}, 0 \leq j \leq m-1\right)$ are Müntz monomials; $\tau_{z}(z \in I)$ denotes the Hankel translation operator of order $\mu$; and $\alpha_{i}, \beta_{j}\left(i, j \in \mathbb{Z}_{+}, 1 \leq i \leq n, 0 \leq j \leq\right.$ $m-1)$ are complex coefficients.

When applied to scattered data interpolation the previous scheme leaves a greater variety of manageable kernels at our disposal, which could be useful in handling mathematical models built upon a class of radial basis functions depending on the order $\mu$ and whose performance is expected to improve by adjusting $\mu$, as it happens with the family of Matérn kernels in [17, Supplement, p. 6]; the examples and numerical experiments exhibited in [14] seem to support this view. Other potential applications of interpolation by Hankel translates of a basis function are in the field of radial basis function neural networks [18-20].

It may be observed that the seminorm in (23) is written in terms of the Hankel transform of the function $f$ (an indirect seminorm) rather than $f$ itself (a direct seminorm). The latter is more convenient for the purpose of obtaining error estimates, however. Motivated by [21], in [22] we expressed the indirect seminorm (23) in two equivalent direct forms, which were referred to as seminorms of type I and type II. Here we want to use type II seminorms to gain a deeper understanding of the spaces $Y_{m}\left(m \in \mathbb{Z}_{+}\right)$. We show that, under rather general conditions on the weight $w$, which are satisfied by those weights giving rise to seminorms of type II, the Zemanian spaces $\mathscr{B}_{\mu}$ and $\mathscr{H}_{\mu}$ are dense in $Y_{m}\left(m \in \mathbb{Z}_{+}\right)$.

1.4. Structure and Notation. This paper is organized as follows. In Section 2 we recall the definition of a seminorm of type II and introduce the notion of strong type II seminorm. Also, we prove that those weights giving rise to type II seminorms are integrable near zero and exhibit polynomial growth at infinity. With the aid of some preliminary lemmas concerning Hankel approximate identities, the density of $\mathscr{B}_{\mu}$ and $\mathscr{H}_{\mu}$ in $Y_{m}\left(m \in \mathbb{Z}_{+}\right)$is finally proved in Section 3 .

Throughout the rest of this paper, the positive real axis will be always denoted by $I$, while $\mu$ will stand for a real number strictly greater than $-1 / 2$, and $C$ will represent a suitable positive constant, depending only on the opportune subscripts (if any), whose value may vary from line to line. Moreover, we shall adhere to the notations $\mathbb{Z}_{+}=\mathbb{N} \cup\{0\}$ for the set of nonnegative integers and $\mathscr{J}_{\mu}(z)=z^{1 / 2} J_{\mu}(z)(z \in I)$ for the function giving the kernel of the Hankel transformation $h_{\mu}$. The following classes of functions will be occasionally used: $\mathscr{C}$, formed by the continuous functions on $I$, and $\mathscr{E}$, consisting of all those infinitely differentiable functions on I. For the operational rules of the Hankel transformation and further properties of the Hankel translation and Hankel convolution that eventually might be required, both in the classical and the generalized senses, the reader is mainly referred to $[3-5,7,23,24]$.

\section{Seminorms of Type II}

Denote by $L_{\mu, c}^{1}$ the class of all those measurable functions $u=$ $u(t)(t \in I)$ such that

$$
\int_{a}^{\infty}|u(t)| t^{\mu+1 / 2} d t<\infty \quad(a>0) .
$$


Definition 2. A seminorm of the form given in (23) is called a type II seminorm provided that

$$
\begin{aligned}
w(t)= & 2 c_{\mu}^{-1} t^{-2 \mu-1} W(t)-t^{-3 \mu-3 / 2}\left(\tau_{t} W\right)(t) \\
& -c_{\mu}^{-1} t^{-\mu-1 / 2} \lim _{z \rightarrow 0+} z^{-\mu-1 / 2} W(z)>0 \quad(t \in I),
\end{aligned}
$$

where

(i) the distribution $W \in \mathscr{H}_{\mu}^{\prime}$ is regular, generated by a continuous function on $I$, such that $W \in L_{\mu}^{\infty}$ and the $\operatorname{limit}_{\lim _{t \rightarrow 0+}} t^{-\mu-1 / 2} W(t)$ exists,

(ii) $h_{\mu}^{\prime} W \in L_{\mu, c}^{1}$,

(iii) $\left(h_{\mu}^{\prime} W\right)(x)=\mathcal{O}\left(x^{\gamma}\right)$ as $x \rightarrow 0+$, where $\gamma+\mu+7 / 2>0$, and

(iv) $\left(h_{\mu}^{\prime} W\right)(x) \leq 0$ a.e. $x \in I$.

If condition $\gamma+\mu+7 / 2>0$ in part (iii) is replaced with the stronger one $\gamma+\mu+3 / 2>0$, then we call (23) a strong type II seminorm.

Example 3 (strong type II seminorm). Set $W(t)=-t^{\mu+1 / 2}$ $e^{-t^{2} / 2}(t \in I)$. The change of variables $t^{2} / 2=x$ leads to

$$
\begin{aligned}
\int_{0}^{\infty}|W(t)| t^{\mu+1 / 2} d t & =\int_{0}^{\infty} e^{-t^{2} / 2} t^{2 \mu+1} d t \\
& =2^{\mu} \int_{0}^{\infty} e^{-x} x^{\mu} d x=2^{\mu} \Gamma(\mu+1)=c_{\mu},
\end{aligned}
$$

so that $W \in L_{\mu}^{1} \subset \mathscr{H}_{\mu}^{\prime}$. Moreover, $\lim _{t \rightarrow 0+} t^{-\mu-1 / 2} W(t)=-1$, $\lim _{t \rightarrow \infty} t^{-\mu-1 / 2} W(t)=0$, and $\left(h_{\mu}^{\prime} W\right)(x)=W(x)(x \in I)$ [25, Equation 8.6(10)]. Thus, $W$ satisfies the strong condition (iii), hence the weak one (with $\gamma=\mu+1 / 2$ ), as well as the remaining conditions (i), (ii), and (iv) in Definition 2. For $\mu=1 / 2$, with the aid of Maple 14, the weight defined by (27) is found to be

$$
w(t)=(2 \pi)^{-1 / 2} t^{-3}\left(1-e^{-2 t^{2}}+2 t^{2}-4 t^{2} e^{-t^{2} / 2}\right) \quad(t \in I),
$$

and the expression in parentheses can be seen to be positive for $t \neq 0$. Consequently, $w(t)>0(t \in I)$.

Theorem 5 later will show that condition (i) in Definition 2 above is somewhat redundant and, at the same time, will shed some light on how to construct weights giving rise to seminorms of type II. The following preliminary result is well known; we include it for the sake of completeness.

Lemma 4. Let $f \in L_{\mu}^{1}$. Then

(i) $h_{\mu} f \in \mathscr{C}$,

(ii) the limit $\lim _{x \rightarrow 0+} x^{-\mu-1 / 2}\left(h_{\mu} f\right)(x)$ exists,

(iii) $\lim _{x \rightarrow \infty} x^{-\mu-1 / 2}\left(h_{\mu} f\right)(x)=0$, and

(iv) $\left\|h_{\mu} f\right\|_{\mu, \infty} \leq c_{\mu}^{-1}\|f\|_{\mu, 1}$, where $c_{\mu}=2^{\mu} \Gamma(\mu+1)$.
Proof. It suffices to observe that

$$
\begin{array}{r}
x^{-\mu-1 / 2}\left(h_{\mu} f\right)(x)=\int_{0}^{\infty} f(t)(x t)^{-\mu} J_{\mu}(x t) t^{\mu+1 / 2} d t \\
(x \in I),
\end{array}
$$

where $f \in L_{\mu}^{1}$ and the function $z^{-\mu} J_{\mu}(z)(z \in I)$ is continuous, with $\sup _{z \in I}\left|z^{-\mu} J_{\mu}(z)\right| \leq c_{\mu}^{-1}$ [26, Equation 9.1.62], $\lim _{z \rightarrow 0+} z^{-\mu} J_{\mu}(z)=c_{\mu}^{-1}$ [26, Equation 9.1.7], and $\lim _{z \rightarrow \infty}$ $z^{-\mu} J_{\mu}(z)=0$ [26, Equation 9.2.1].

The space $\mathscr{E}_{\mu}$ consists of all those $u \in \mathscr{E}$ such that

$$
\kappa_{\mu, l, r}(u)=\sup _{z \in] 0, l[}\left|\left(z^{-1} D\right)^{r} z^{-\mu-1 / 2} u(z)\right|<\infty
$$

$$
\left(l \in \mathbb{N}, r \in \mathbb{Z}_{+}\right) \text {. }
$$

Endowed with the topology generated by the family of seminorms $\left\{\kappa_{\mu, l, r}\right\}_{(l, r) \in \mathbb{N} \times \mathbb{Z}_{+}}, \mathscr{E}_{\mu}$ becomes a Fréchet space [23, Proposition 4.3]. As usual, its dual space will be denoted by $\mathscr{E}_{\mu}^{\prime}$. The inclusions $\mathscr{B}_{\mu} \subset \mathscr{H}_{\mu} \subset \mathscr{E}_{\mu}$ being dense, we have $\mathscr{E}_{\mu}^{\prime} \subset \mathscr{H}_{\mu}^{\prime} \subset \mathscr{B}_{\mu}^{\prime}[23$, Proposition 4.4].

Theorem 5. Let $f \in L_{\mu, c}^{1}$ and assume $f(t)=\mathcal{O}\left(t^{\gamma}\right)$ as $t \rightarrow$ $0+$, for some $\gamma+\mu+3 / 2>0$. Then

(i) $f \in \mathscr{H}_{\mu}^{\prime}$,

(ii) $h_{\mu}^{\prime} f \in \mathscr{C}$,

(iii) the limit $\lim _{x \rightarrow 0+} x^{-\mu-1 / 2}\left(h_{\mu}^{\prime} f\right)(x)$ exists, and

(iv) some $r \in \mathbb{N}$ is such that

$$
\left(h_{\mu}^{\prime} f\right)(x)=\mathcal{O}\left(x^{2 r+\mu+1 / 2}\right) \text { as } x \longrightarrow \infty \text {. }
$$

Proof. From the condition $f(t)=\mathcal{O}\left(t^{\gamma}\right)$ as $t \rightarrow 0+$, it follows that

$$
|f(t)| \leq C t^{\gamma} \quad(0<t<a)
$$

for some $a, C>0$. Define $g(t)=0(0<t<a), g(t)=$ $f(t)(t \geq a)$. Clearly,

$$
f(t)=g(t)+(f-g)(t) \quad(t \in I) .
$$

As $f \in L_{\mu, c}^{1}$, we have $g \in L_{\mu}^{1}$, and hence $g$ defines a distribution in $\mathscr{H}_{\mu}^{\prime}$.

We claim that $f-g \in \mathscr{E}_{\mu}^{\prime}$. First we observe that $(f-g)(t)=$ $f(t)(0<t<a)$ while $(f-g)(t)=0(t \geq a)$, so that, from (33),

$$
B=\int_{0}^{\infty}|(f-g)(t)| t^{\mu+1 / 2} d t \leq C \int_{0}^{a} t^{\gamma+\mu+1 / 2} d t<\infty,
$$

because $\gamma+\mu+3 / 2>0$. Denoting by $l$ the least positive integer greater than $a$, we may then write

$$
|\langle f-g, \phi\rangle|=\left|\int_{0}^{\infty}(f-g)(t) \phi(t) d t\right| \leq B \kappa_{\mu, l, 0}(\phi)
$$

$$
\left(\phi \in \mathscr{E}_{\mu}\right) \text {, }
$$


which proves our claim. In particular, $f-g \in \mathscr{H}_{\mu}^{\prime}$, and from (34) we conclude that $f \in \mathscr{H}_{\mu}^{\prime}$.

Since $f-g \in \mathscr{E}_{\mu}^{\prime}$, [23, Propositions 4.5 and 4.6] yield $b, C>0, r \in \mathbb{N}$ for which

$$
\left|x^{-\mu-1 / 2} h_{\mu}^{\prime}(f-g)(x)\right| \leq C x^{2 r} \quad(x>b) .
$$

And since $g \in L_{\mu}^{1}$, Lemma 4 entails that $\lim _{x \rightarrow \infty} x^{-\mu-1 / 2}$ $\left(h_{\mu}^{\prime} g\right)(x)=0$. Therefore, for some $c>0$,

$$
\left|x^{-\mu-1 / 2}\left(h_{\mu}^{\prime} g\right)(x)\right| \leq C x^{2 r} \quad(x>c) .
$$

A combination of (37) and (38) establishes (32).

Applying [7, Lemma 3.2] to $f-g \in \mathscr{E}_{\mu}^{\prime} \subset \mathscr{E}$, we find

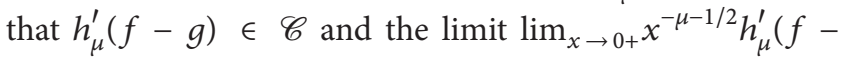
$g)(x)$ exists. Moreover, as $g \in L_{\mu}^{1}$, from Lemma 4 we obtain

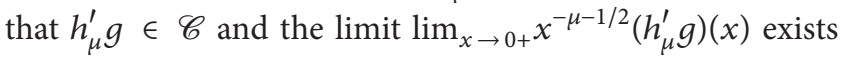
as well. This shows that $h_{\mu}^{\prime} f \in \mathscr{C}$ and the $\operatorname{limit}_{x \rightarrow 0+}$ $x^{-\mu-1 / 2}\left(h_{\mu}^{\prime} f\right)(x)$ exists. The proof is thus complete.

Corollary 6. Assume $f \in L_{\mu, c}^{1}$ and $f(t)=\mathcal{O}\left(t^{\gamma}\right)$ as $t \rightarrow 0+$ for some $\gamma+\mu+3 / 2>0$, so that, by Theorem $5, f \in \mathscr{H}_{\mu}^{\prime}$. If $f(t) \leq 0$ a.e. $t \in I$ and $W=h_{\mu}^{\prime} f \in L_{\mu}^{\infty}$, then $W$ satisfies conditions (i) to (iv) of Definition 2.

Proof. From Theorem 5 we find that $W \in \mathscr{H}_{\mu}^{\prime} \cap \mathscr{C}$ and the limit $\lim _{t \rightarrow 0+} t^{-\mu-1 / 2} W(t)$ exists. This yields condition (i) in Definition 2. Since $f=h_{\mu}^{\prime} W$, conditions (ii) to (iv) are trivially satisfied.

Proposition 7. If the weight $w$ gives rise to a type II seminorm as in Definition 2, then $w \in L_{\mu, l}^{1}$ and $w(t)=\mathscr{O}\left(t^{\gamma}\right)$ as $t \rightarrow \infty$ for some $\gamma \in \mathbb{R}$.

Proof. Associate $W$ to $w$ as in Definition 2, and let $f=h_{\mu}^{\prime} W$. From (5) and (8) we have

$$
\begin{aligned}
\left|\left(\tau_{t} W\right)(t)\right| & =\left|\int_{0}^{\infty} W(z) D_{\mu}(t, t, z) d z\right| \\
& \leq \int_{0}^{\infty}\left|z^{-\mu-1 / 2} W(z)\right| D_{\mu}(t, t, z) z^{\mu+1 / 2} d z \\
& \leq\|W\|_{\mu, \infty} \int_{0}^{\infty} D_{\mu}(t, t, z) z^{\mu+1 / 2} d z \\
& =c_{\mu}^{-1}\|W\|_{\mu, \infty} t^{2 \mu+1} \quad(t \in I),
\end{aligned}
$$

or

$$
\left|t^{-2 \mu-1}\left(\tau_{t} W\right)(t)\right| \leq c_{\mu}^{-1}\|W\|_{\mu, \infty} \quad(t \in I) .
$$

Consequently, there exists $C>0$ for which

$$
\begin{aligned}
w(t)= & c_{\mu}^{-1} t^{-\mu-1 / 2} \mid 2 t^{-\mu-1 / 2} W(t)-c_{\mu} t^{-2 \mu-1}\left(\tau_{t} W\right)(t) \\
& \quad-\lim _{z \rightarrow 0+} z^{-\mu-1 / 2} W(z) \mid \\
& \leq c_{\mu}^{-1} t^{-\mu-1 / 2}\left(2\|W\|_{\mu, \infty}+\|W\|_{\mu, \infty}+\|W\|_{\mu, \infty}\right) \\
& =C t^{-\mu-1 / 2} \quad(t \in I) .
\end{aligned}
$$

This establishes the proposition.

\section{Density Results}

In this section we prove two density results for weights $w=$ $w(x)>0(x \in I)$ satisfying the conditions in the thesis of Proposition 7; namely, the Zemanian spaces $\mathscr{B}_{\mu}$ and $\mathscr{H}_{\mu}$ are dense in $Y_{m}\left(m \in \mathbb{Z}_{+}\right)$. Both of these results will therefore hold true for spaces endowed with type II seminorms. In this way, direct seminorms allow us to establish direct counterparts of [14, Theorem 2.23], where the space $\left\{u \in Y_{m}\right.$ : $\left.h_{\mu}^{\prime} u \in \mathscr{B}_{\mu}\right\}$ and hence $\left\{u \in Y_{m}: h_{\mu}^{\prime} u \in \mathscr{H}_{\mu}\right\}$ were shown to be dense in $Y_{m}\left(m \in \mathbb{Z}_{+}\right)$.

Proposition 8. If $w \in L_{\mu, l}^{1}$ and there exists $\gamma \in \mathbb{R}$ such that $w(x)=\mathcal{O}\left(x^{\gamma}\right)$ as $x \rightarrow \infty$, then $\mathscr{H}_{\mu} \subset Y_{m}\left(m \in \mathbb{Z}_{+}\right)$.

Proof. For $m=0$, this is [14, Theorem 2.12]; the proof below runs along similar lines, and we include it for completeness. The following operational rule of the Hankel transformation will be used [24, Equation 5.4(5)]:

$$
h_{\mu}\left(S_{\mu} \varphi\right)(t)=-t^{2}\left(h_{\mu} \varphi\right)(t) \quad\left(t \in I, \varphi \in \mathscr{H}_{\mu}\right) .
$$

From the hypothesis, there exist $a, C>0$ such that $w(x) \leq$ $C x^{\gamma}(x>a)$. Fix $\varphi \in \mathscr{H}_{\mu}$. Then,

$$
\begin{aligned}
& \int_{0}^{\infty}\left|\left(h_{\mu} S_{\mu}^{m} \varphi\right)(t)\right|^{2} w(t) t^{\mu+1 / 2} d t \\
& \quad=\left\{\int_{0}^{a}+\int_{a}^{\infty}\right\}\left|\left(h_{\mu} S_{\mu}^{m} \varphi\right)(t)\right|^{2} w(t) t^{\mu+1 / 2} d t .
\end{aligned}
$$

The first integral on the right-hand side of this identity is finite because $w \in L_{\mu, l}^{1}$ :

$$
\begin{aligned}
& \int_{0}^{a}\left|\left(h_{\mu} S_{\mu}^{m} \varphi\right)(t)\right|^{2} w(t) t^{\mu+1 / 2} d t \\
& =\int_{0}^{a} t^{4 m}\left|\left(h_{\mu} \varphi\right)(t)\right|^{2} w(t) t^{\mu+1 / 2} d t \\
& =\int_{0}^{a} t^{4 m+2 \mu+1}\left|t^{-\mu-1 / 2}\left(h_{\mu} \varphi\right)(t)\right|^{2} w(t) t^{\mu+1 / 2} d t \\
& \leq a^{4 m+2 \mu+1} \sup _{z \in I}\left|z^{-\mu-1 / 2}\left(h_{\mu} \varphi\right)(z)\right|^{2} \\
& \quad \times \int_{0}^{a} w(t) t^{\mu+1 / 2} d t<\infty .
\end{aligned}
$$


On the other hand,

$$
\begin{aligned}
& \int_{a}^{\infty}\left|\left(h_{\mu} S_{\mu}^{m} \varphi\right)(t)\right|^{2} w(t) t^{\mu+1 / 2} d t \\
& =\int_{a}^{\infty} t^{4 m}\left|\left(h_{\mu} \varphi\right)(t)\right|^{2} w(t) t^{\mu+1 / 2} d t \\
& =\int_{a}^{\infty} t^{4 m-2 n+2 \mu+1}\left|t^{n-\mu-1 / 2}\left(h_{\mu} \varphi\right)(t)\right|^{2} \\
& \quad \times w(t) t^{\mu+1 / 2} d t \\
& \leq C \sup _{z \in I}\left|z^{n-\mu-1 / 2}\left(h_{\mu} \varphi\right)(z)\right|^{2} \\
& \quad \times \int_{a}^{\infty} t^{4 m-2 n+\gamma+3 \mu+3 / 2} d t<\infty,
\end{aligned}
$$

provided that $2 n>4 m+\gamma+3 \mu+5 / 2$.

Lemma 9. Let $\psi \in L_{\mu}^{1}$ satisfy $\psi(x) \geq 0(x \in I)$ and $\int_{0}^{\infty} \psi(x) x^{\mu+1 / 2} d x=c_{\mu}$, where $c_{\mu}=2^{\mu} \Gamma(\mu+1)$. Define $\psi_{k}(x)=$ $k^{\mu+3 / 2} \psi(k x)(x \in I, k \in \mathbb{N})$. If $\phi \in L_{\mu}^{\infty}$ is continuous at $x_{0} \in I$, then

$$
\lim _{k \rightarrow \infty}\left(\phi \# \psi_{k}\right)\left(x_{0}\right)=\phi\left(x_{0}\right)
$$

If, moreover, $x^{-\mu-1 / 2} \phi(x)(x \in I)$ is uniformly continuous, then

$$
\lim _{k \rightarrow \infty}\left\|\phi \# \psi_{k}-\phi\right\|_{\mu, \infty}=0
$$

Proof. Note that

$$
\begin{gathered}
\left\|\psi_{k}\right\|_{\mu, 1}=c_{\mu} \quad(k \in \mathbb{N}), \\
\lim _{k \rightarrow \infty} \int_{\delta}^{\infty} \psi_{k}(x) x^{\mu+1 / 2} d x=0 \quad(\delta>0) .
\end{gathered}
$$

Put $\varphi(x)=x^{-\mu-1 / 2} \phi(x)(x \in I)$. Since $\varphi$ is continuous at $x_{0}$, given $\varepsilon>0$, there exists $\delta=\delta\left(x_{0}, \varepsilon\right)>0$ such that $\left|x-x_{0}\right|<\delta$ implies $\left|\varphi(x)-\varphi\left(x_{0}\right)\right|<\varepsilon$. In view of (4), (5), and (8), we are allowed to write

$$
\begin{aligned}
& \left(\phi \# \psi_{k}\right)\left(x_{0}\right)-\phi\left(x_{0}\right) \\
& =\int_{0}^{\infty} \int_{0}^{\infty}\left[\varphi(x)-\varphi\left(x_{0}\right)\right] \\
& \quad \times \psi_{k}(y) D_{\mu}\left(x_{0}, x, y\right) x^{\mu+1 / 2} d x d y \\
& =\left\{\int_{0}^{\delta}+\int_{\delta}^{\infty}\right\} \int_{0}^{\infty}\left[\varphi(x)-\varphi\left(x_{0}\right)\right] \\
& \quad \times \psi_{k}(y) D_{\mu}\left(x_{0}, x, y\right) x^{\mu+1 / 2} d x d y .
\end{aligned}
$$

From (7) we find that $D_{\mu}\left(x_{0}, x, y\right) \neq 0(0<y<\delta)$ forces $\left|x-x_{0}\right|<\delta$; in this case, we have $\left|\varphi(x)-\varphi\left(x_{0}\right)\right|<\varepsilon$. Along with (48) and (8), this leads us to

$$
\begin{aligned}
& \left|\int_{0}^{\delta} \int_{0}^{\infty}\left[\varphi(x)-\varphi\left(x_{0}\right)\right] \psi_{k}(y) D_{\mu}\left(x_{0}, x, y\right) x^{\mu+1 / 2} d x d y\right| \\
& \quad \leq \varepsilon \int_{0}^{\delta} \int_{0}^{\infty} \psi_{k}(y) D_{\mu}\left(x_{0}, x, y\right) x^{\mu+1 / 2} d x d y \\
& \quad \leq \varepsilon c_{\mu}^{-1} \int_{0}^{\delta} \psi_{k}(y)\left(x_{0} y\right)^{\mu+1 / 2} d y \\
& \quad \leq \varepsilon x_{0}^{\mu+1 / 2}
\end{aligned}
$$

On the other hand, in view of (49) and (8), there exists $k_{0} \in \mathbb{N}$ for which

$$
\begin{aligned}
& \left|\int_{\delta}^{\infty} \int_{0}^{\infty}\left[\varphi(x)-\varphi\left(x_{0}\right)\right] \psi_{k}(y) D_{\mu}\left(x_{0}, x, y\right) x^{\mu+1 / 2} d x d y\right| \\
& \quad \leq 2\|\phi\|_{\mu, \infty} \int_{\delta}^{\infty} \int_{0}^{\infty} \psi_{k}(y) D_{\mu}\left(x_{0}, x, y\right) x^{\mu+1 / 2} d x d y \\
& \quad=2 c_{\mu}^{-1}\|\phi\|_{\mu, \infty} \int_{\delta}^{\infty} \psi_{k}(y)\left(x_{0} y\right)^{\mu+1 / 2} d y \\
& \quad<\varepsilon x_{0}^{\mu+1 / 2} \quad\left(k \in \mathbb{N}, k \geq k_{0}\right) .
\end{aligned}
$$

At this point, (46) follows from (50), (51), and (52). Moreover, if $\varphi$ is uniformly continuous on $I$, then $\delta$ and hence $k_{0}$ in the previous argument do not depend on $x_{0}$, so that (47) holds.

Lemma 10. Assume $w(x)=\mathcal{O}\left(x^{\gamma}\right)$ as $x \rightarrow \infty$ for some $\gamma \in$ $\mathbb{R}$. Let $\psi \in \mathscr{H}_{\mu}$ satisfy $\psi(t) \geq 0(t \in I)$ and $\int_{0}^{\infty} \psi(t) t^{\mu+1 / 2} d t=$ $c_{\mu}$. Define $\psi_{k}(t)=k^{\mu+3 / 2} \psi(k t)(t \in I, k \in \mathbb{N})$. Given $f \in \mathscr{H}_{\mu}$ and $\varepsilon>0$, there exists $a>0$ such that

$$
\int_{a}^{\infty}\left|\left(f \# \psi_{k}\right)(t)\right|^{2} w(t) t^{\mu+1 / 2} d t<\varepsilon \quad(k \in \mathbb{N}) .
$$

Proof. Fix $r, k \in \mathbb{N}$ and $x \in I$. Since $\psi \in L_{\mu}^{1}$, some $b>0$ is such that

$$
\int_{b}^{\infty} \psi(t) t^{\mu+1 / 2} d t \leq \frac{1}{\left(1+x^{2}\right)^{r}}
$$

Clearly,

$$
\begin{aligned}
\left|\left(f \# \psi_{k}\right)(x)\right|= & \left|\int_{0}^{\infty}\left(\tau_{x} f\right)(t) \psi_{k}(t) d t\right| \\
= & \left|\left\{\int_{0}^{b}+\int_{b}^{\infty}\right\}\left(\tau_{x} f\right)(t) \psi_{k}(t) d t\right| \\
\leq & \left|\int_{0}^{b}\left(\tau_{x} f\right)(t) \psi_{k}(t) d t\right| \\
& +\left|\int_{b}^{\infty}\left(\tau_{x} f\right)(t) \psi_{k}(t) d t\right| .
\end{aligned}
$$


As in the proof of Proposition 7, for the second integral on the right-hand side of (55) we arrive at

$$
\begin{aligned}
& \left|\int_{b}^{\infty}\left(\tau_{x} f\right)(t) \psi_{k}(t) d t\right| \\
& \quad \leq c_{\mu}^{-1}\|f\|_{\mu, \infty} x^{\mu+1 / 2} \int_{b}^{\infty} \psi_{k}(t) t^{\mu+1 / 2} d t \\
& \quad=C x^{\mu+1 / 2} \int_{k b}^{\infty} \psi(t) t^{\mu+1 / 2} d t \\
& \quad \leq C x^{\mu+1 / 2} \int_{b}^{\infty} \psi(t) t^{\mu+1 / 2} d t \leq C \frac{x^{\mu+1 / 2}}{\left(1+x^{2}\right)^{r}} .
\end{aligned}
$$

In order to estimate the first integral on the right-hand side of (55), fix $t \in] 0, b$ [ and apply the self-reciprocity of $h_{\mu}$ on $\mathscr{H}_{\mu}$, along with (42) and (14), to get

$$
\begin{aligned}
& \left|t^{-\mu-1 / 2}\left(\tau_{x} f\right)(t)\right| \\
& =\frac{x^{\mu+1 / 2}}{\left(1+x^{2}\right)^{r}} \mid x^{-\mu-1 / 2} h_{\mu}\left[\left(1-S_{\mu, z}\right)^{r}\right. \\
& \left.\quad \times(z t)^{-\mu} J_{\mu}(z t)\left(h_{\mu} f\right)(z)\right](x) \mid \\
& \leq c_{\mu}^{-1} \frac{x^{\mu+1 / 2}}{\left(1+x^{2}\right)^{r}} \int_{0}^{\infty} \mid\left(1-S_{\mu, z}\right)^{r}(z t)^{-\mu} \\
& \times J_{\mu}(z t)\left(h_{\mu} f\right)(z) \mid z^{\mu+1 / 2} d z .
\end{aligned}
$$

For any fixed $z \in I$ and suitable constants $a_{n, i}(n, i \in \mathbb{N}, 0 \leq$ $i \leq n \leq r)$, we may write

$$
\begin{aligned}
(1 & \left.-S_{\mu, z}\right)^{r}(z t)^{-\mu} J_{\mu}(z t)\left(h_{\mu} f\right)(z) \\
= & \sum_{n=0}^{r}(-1)^{n}\left(\begin{array}{l}
r \\
n
\end{array}\right) S_{\mu, z}^{n}(z t)^{-\mu} J_{\mu}(z t)\left(h_{\mu} f\right)(z) \\
= & z^{\mu+1 / 2} \sum_{n=0}^{r}(-1)^{n}\left(\begin{array}{l}
r \\
n
\end{array}\right) z^{-\mu-1 / 2} S_{\mu, z}^{n}(z t)^{-\mu} J_{\mu}(z t)\left(h_{\mu} f\right)(z) \\
= & z^{\mu+1 / 2} \sum_{n=0}^{r}(-1)^{n}\left(\begin{array}{l}
r \\
n
\end{array}\right) \\
& \times \sum_{i=0}^{n} a_{n, i} z^{2 i}\left(z^{-1} D_{z}\right)^{n+i}(z t)^{-\mu} J_{\mu}(z t) z^{-\mu-1 / 2}\left(h_{\mu} f\right)(z),
\end{aligned}
$$

with

$$
\begin{gathered}
z^{2 i+\mu+1 / 2}\left(z^{-1} D_{z}\right)^{n+i}(z t)^{-\mu} J_{\mu}(z t) z^{-\mu-1 / 2}\left(h_{\mu} f\right)(z) \\
=\sum_{j=0}^{n+i}\left(\begin{array}{c}
n+i \\
j
\end{array}\right)\left[\left(z^{-1} D_{z}\right)^{j}(z t)^{-\mu} J_{\mu}(z t)\right]
\end{gathered}
$$

$$
\begin{aligned}
& \times\left[z^{2 i+\mu+1 / 2}\left(z^{-1} D_{z}\right)^{n+i-j} z^{-\mu-1 / 2}\left(h_{\mu} f\right)(z)\right] \\
= & \sum_{j=0}^{n+i}\left(\begin{array}{c}
n+i \\
j
\end{array}\right)\left[\left(-t^{2}\right)^{j}(z t)^{-\mu-j} J_{\mu+j}(z t)\right] \\
& \times\left[z^{2 i+\mu+1 / 2}\left(z^{-1} D_{z}\right)^{n+i-j} z^{-\mu-1 / 2}\left(h_{\mu} f\right)(z)\right] .
\end{aligned}
$$

Since $\sup _{y \in I}\left|y^{-v} J_{\nu}(y)\right| \leq c_{\nu}^{-1}(\nu \geq-1 / 2)$ and $h_{\mu} f \in \mathscr{H}_{\mu}$, we find that the right-hand side of (59) is bounded by $\left(1+t^{2}\right)^{2 r}$ times an $L_{\mu}^{1}$ function of $z \in I$. It then follows from (57) that

$$
\left|t^{-\mu-1 / 2}\left(\tau_{x} f\right)(t)\right| \leq C \frac{x^{\mu+1 / 2}}{\left(1+x^{2}\right)^{r}}\left(1+t^{2}\right)^{2 r} \quad(t \in] 0, b[) .
$$

Consequently,

$$
\begin{aligned}
& \left|\int_{0}^{b}\left(\tau_{x} f\right)(t) \psi_{k}(t) d t\right| \\
& \quad \leq \int_{0}^{b}\left|t^{-\mu-1 / 2}\left(\tau_{x} f\right)(t)\right| \psi_{k}(t) t^{\mu+1 / 2} d t \\
& \quad \leq C \frac{x^{\mu+1 / 2}}{\left(1+x^{2}\right)^{r}} \int_{0}^{b}\left(1+t^{2}\right)^{2 r} \psi_{k}(t) t^{\mu+1 / 2} d t .
\end{aligned}
$$

A change of variables leads to

$$
\begin{aligned}
& \int_{0}^{b}\left(1+t^{2}\right)^{2 r} \psi_{k}(t) t^{\mu+1 / 2} d t \\
& \quad=\int_{0}^{k b}\left(1+\frac{t^{2}}{k^{2}}\right)^{2 r} \psi(t) t^{\mu+1 / 2} d t \\
& \quad \leq \int_{0}^{\infty}\left(1+\frac{t^{2}}{k^{2}}\right)^{2 r} \psi(t) t^{\mu+1 / 2} d t .
\end{aligned}
$$

The sequence of integrals on the right-hand side of (62) converges to $c_{\mu}$ as $k \rightarrow \infty$ and is therefore bounded. This follows from Lebesgue's monotone convergence theorem applied to

$$
\varphi_{n}(t)=\left(1+\frac{t^{2}}{n^{2}}\right)^{2 r} \psi(t) \quad(t \in I, n \in \mathbb{N}) .
$$

Indeed, the sequence $\left\{\varphi_{n}\right\}_{n \in \mathbb{N}}$ is nonincreasing and $\varphi_{1}(t)=$ $\left(1+t^{2}\right)^{2 r} \psi(t) \in \mathscr{H}_{\mu} \subset L_{\mu}^{1}$, because $\psi \in \mathscr{H}_{\mu}$ and even polynomials are multipliers of $\mathscr{H}_{\mu}$ [24, Lemma 5.3.1]. Thus, from (61),

$$
\left|\int_{0}^{b}\left(\tau_{x} f\right)(t) \psi_{k}(t) d t\right| \leq C \frac{x^{\mu+1 / 2}}{\left(1+x^{2}\right)^{r}} .
$$

Plugging (56) and (64) into (55) finally yields

$$
\left|\left(f \# \psi_{k}\right)(x)\right|^{2} \leq C \frac{x^{2 \mu+1}}{\left(1+x^{2}\right)^{2 r}} \quad(x \in I) .
$$


The hypothesis that $w(x)=\mathcal{O}\left(x^{\gamma}\right)$ as $x \rightarrow \infty$ furnishes $s, C>0$ for which $w(x) \leq C x^{\gamma}(x>s)$. Combining this estimate with (65), we obtain

$$
\int_{a}^{\infty}\left|\left(f \# \psi_{k}\right)(x)\right|^{2} w(x) x^{\mu+1 / 2} d x \leq C \int_{a}^{\infty} \frac{x^{\gamma+3 \mu+3 / 2}}{\left(1+x^{2}\right)^{2 r}} d x<\infty
$$

provided that $r \in \mathbb{N}$ is chosen so that $4 r>\gamma+3 \mu+5 / 2$. This entails

$$
\lim _{a \rightarrow \infty} \int_{a}^{\infty}\left|\left(f \# \psi_{k}\right)(x)\right|^{2} w(x) x^{\mu+1 / 2} d x=0
$$

uniformly in $k \in \mathbb{N}$ and completes the proof.

Theorem 11. Let $m \in \mathbb{Z}_{+}$, and let $w \in L_{\mu, l}^{1}$ satisfy $w(x)=$ $\mathcal{O}\left(x^{\gamma}\right)$ as $x \rightarrow \infty$ for some $\gamma \in \mathbb{R}$. The space $\mathscr{B}_{\mu}$ is a subspace of $Y_{m}$. Furthermore, $\mathscr{B}_{\mu}$ is a dense subspace of $Y_{m}$, in the following sense: given $f \in Y_{m}$ and $\varepsilon>0$, there exists $u \in \mathscr{B}_{\mu}$ such that $|f-u|_{m}<\varepsilon$.

Proof. From Proposition 8, $\mathscr{H}_{\mu} \subset Y_{m}$. As $\mathscr{B}_{\mu} \subset \mathscr{H}_{\mu}, \mathscr{B}_{\mu}$ is a subspace of $Y_{m}$.

Take $\psi \in \mathscr{H}_{\mu}$ such that $\psi(t) \geq 0(t \in I), \int_{0}^{\infty} \psi(t) t^{\mu+1 / 2}$ $d t=c_{\mu}$, and $h_{\mu} \psi \in \mathscr{B}_{\mu}$. For each $k \in \mathbb{N}$, define $\psi_{k}(t)=$ $k^{\mu+3 / 2} \psi(k t)(t \in I)$. Let $f \in Y_{m}$ and $\varepsilon>0$. From [14, Theorem 2.23], the set $\left\{u \in Y_{m}: h_{\mu}^{\prime} u \in \mathscr{B}_{\mu}\right\}$ is dense in $Y_{m}$; therefore, we may assume that $h_{\mu}^{\prime} f \in \mathscr{B}_{\mu}$. Set $g_{k}(t)=$ $\left(h_{\mu}^{\prime} f \# \psi_{k}\right)(t)(t \in I, k \in \mathbb{N})$. Then $g_{k} \in \mathscr{H}_{\mu}(k \in \mathbb{N})$, and Lemma 10, applied to the weight $v(t)=t^{4 m} w(t)(t \in I)$, gives $a>0$ such that

$$
\begin{aligned}
& \int_{a}^{\infty}\left|\left(h_{\mu}^{\prime} f\right)(t)\right|^{2} w(t) t^{4 m+\mu+1 / 2} d t=0, \\
& \int_{a}^{\infty}\left|g_{k}(t)\right|^{2} w(t) t^{4 m+\mu+1 / 2} d t<\frac{\varepsilon}{2} \quad(k \in \mathbb{N}) .
\end{aligned}
$$

From (68) and Minkowski's inequality,

$$
\begin{aligned}
& \int_{0}^{\infty}\left|\left(h_{\mu}^{\prime} f-g_{k}\right)(t)\right|^{2} w(t) t^{4 m+\mu+1 / 2} d t \\
& =\int_{0}^{a}\left|t^{-\mu-1 / 2}\left(h_{\mu}^{\prime} f-g_{k}\right)(t)\right|^{2} w(t) t^{4 m+3 \mu+3 / 2} d t \\
& \quad+\int_{a}^{\infty}\left|\left(h_{\mu}^{\prime} f-g_{k}\right)(t)\right|^{2} w(t) t^{4 m+\mu+1 / 2} d t \\
& \leq\left\|h_{\mu}^{\prime} f-g_{k}\right\|_{\mu, \infty}^{2} \int_{0}^{a} w(t) t^{4 m+3 \mu+3 / 2} d t
\end{aligned}
$$

$$
\begin{gathered}
+\left[\left\{\int_{a}^{\infty}\left|\left(h_{\mu}^{\prime} f\right)(t)\right|^{2} w(t) t^{4 m+\mu+1 / 2} d t\right\}^{1 / 2}\right. \\
\left.+\left\{\int_{a}^{\infty}\left|g_{k}(t)\right|^{2} w(t) t^{4 m+\mu+1 / 2} d t\right\}^{1 / 2}\right]^{2} \\
<\left\|h_{\mu}^{\prime} f-g_{k}\right\|_{\mu, \infty}^{2} \int_{0}^{a} w(t) t^{4 m+3 \mu+3 / 2} d t+\frac{\varepsilon}{2} .
\end{gathered}
$$

Note that

$$
\int_{0}^{a} w(t) t^{4 m+3 \mu+3 / 2} d t<\infty,
$$

because $w \in L_{\mu, l}^{1}$. Moreover, since $h_{\mu}^{\prime} f \in \mathscr{B}_{\mu}$, we have that $h_{\mu}^{\prime} f \in L_{\mu}^{\infty}$ and $t^{-\mu-1 / 2}\left(h_{\mu}^{\prime} f\right)(t)(t \in I)$ is uniformly continuous. Apply then Lemma 9 to obtain $k \in \mathbb{N}$ satisfying

$$
\left\|h_{\mu}^{\prime} f-g_{k}\right\|_{\mu, \infty}^{2}<\frac{\varepsilon}{2 \int_{0}^{a} w(t) t^{4 m+3 \mu+3 / 2} d t} .
$$

Hence, for that $k$,

$$
\int_{0}^{\infty}\left|\left(h_{\mu}^{\prime} f-g_{k}\right)(t)\right|^{2} w(t) t^{4 m+\mu+1 / 2} d t<\varepsilon .
$$

Finally, define $u \in \mathscr{H}_{\mu}^{\prime}$ through

$$
\begin{aligned}
u(t) & =\left(h_{\mu}^{\prime} g_{k}\right)(t)=h_{\mu}^{\prime}\left(h_{\mu}^{\prime} f \# \psi_{k}\right)(t) \\
& =t^{-\mu-1 / 2} f(t)\left(h_{\mu} \psi_{k}\right)(t) \quad(t \in I),
\end{aligned}
$$

where the exchange formula (15) has been used. Since $h_{\mu} \psi \in$ $\mathscr{B}_{\mu}$, so does $\left(h_{\mu} \psi_{k}\right)(t)=k^{\mu+1 / 2}\left(h_{\mu} \psi\right)(t / k)(t \in I)[25$, Equation 8.1(2)]. And since $h_{\mu}^{\prime} f \in \mathscr{B}_{\mu} \subset \mathscr{H}_{\mu}$, we have $f \in \mathscr{H}_{\mu} \subset \mathscr{E}_{\mu}$. Thus $u \in \mathscr{B}_{\mu}$, with

$$
\begin{aligned}
|f-u|_{m}^{2} & =\int_{0}^{\infty}\left|h_{\mu}^{\prime} S_{\mu}^{m}(f-u)(t)\right|^{2} w(t) t^{\mu+1 / 2} d t \\
& =\int_{0}^{\infty}\left|\left(h_{\mu}^{\prime} f-g_{k}\right)(t)\right|^{2} w(t) t^{4 m+\mu+1 / 2} d t<\varepsilon,
\end{aligned}
$$

as required.

Corollary 12. Let $m \in \mathbb{Z}_{+}$, and let $w \in L_{\mu, l}^{1}$ satisfy $w(x)=$ $\mathcal{O}\left(x^{\gamma}\right)$ as $x \rightarrow \infty$ for some $\gamma \in \mathbb{R}$. The space $\mathscr{H}_{\mu}$ is a dense subspace of $Y_{m}$, in the following sense: given $f \in Y_{m}^{\mu}$ and $\varepsilon>0$, there exists $u \in \mathscr{H}_{\mu}$ such that $|f-u|_{m}<\varepsilon$.

Proof. It suffices to recall that $\mathscr{B}_{\mu} \subset \mathscr{H}_{\mu}$ and apply Theorem 11.

\section{Acknowledgments}

Cristian Arteaga is supported by a 2011 CajaCanarias Research Grant for Postgraduates. Both authors are partially supported by MICINN-FEDER Grant MTM2011-28781 (Spain). 


\section{References}

[1] A. H. Zemanian, "A distributional Hankel transformation," SIAM Journal on Applied Mathematics, vol. 14, pp. 561-576, 1966.

[2] A. H. Zemanian, "The Hankel transformation of certain distributions of rapid growth," SIAM Journal on Applied Mathematics, vol. 14, pp. 678-690, 1966.

[3] I. I. Hirschman, Jr., "Variation diminishing Hankel transforms," Journal d'Analyse Mathématique, vol. 8, pp. 307-336, 1961.

[4] F. M. Cholewinski, "A Hankel convolution complex inversion theory," Memoirs of the American Mathematical Society, vol. 58, p. 67, 1965.

[5] D. T. Haimo, "Integral equations associated with Hankel convolutions," Transactions of the American Mathematical Society, vol. 116, pp. 330-375, 1965.

[6] J. de Sousa Pinto, "A generalised Hankel convolution," SIAM Journal on Mathematical Analysis, vol. 16, no. 6, pp. 1335-1346, 1985.

[7] I. Marrero and J. J. Betancor, "Hankel convolution of generalized functions," Rendiconti di Matematica e delle sue Applicazioni, vol. 15, no. 3, pp. 351-380, 1995.

[8] C. A. Micchelli, "Interpolation of scattered data: distance matrices and conditionally positive definite functions," Constructive Approximation, vol. 2, no. 1, pp. 11-22, 1986.

[9] W. Madych and S. Nelson, "Multivariate interpolation: a variational theory," Unpublished Manuscript, 1983.

[10] W. R. Madych and S. A. Nelson, "Multivariate interpolation and conditionally positive definite functions," Approximation Theory and Its Applications, vol. 4, no. 4, pp. 77-89, 1988.

[11] W. R. Madych and S. A. Nelson, "Multivariate interpolation and conditionally positive definite functions-II," Mathematics of Computation, vol. 54, no. 189, pp. 211-230, 1990.

[12] W. Light and H. Wayne, "Spaces of distributions, interpolation by translates of a basis function and error estimates," Numerische Mathematik, vol. 81, no. 3, pp. 415-450, 1999.

[13] R. Schaback and Z. Wu, "Operators on radial functions," Journal of Computational and Applied Mathematics, vol. 73, no. 1-2, pp. 257-270, 1996.

[14] C. Arteaga and I. Marrero, "A scheme for interpolation by Hankel translates of a basis function," Journal of Approximation Theory, vol. 164, no. 12, pp. 1540-1576, 2012.

[15] E. M. Stein and G. Weiss, Introduction to Fourier Analysis on Euclidean Spaces, Princeton University Press, Princeton, NJ, USA, 1971.

[16] G. Gigante, “Transference for hypergroups," Collectanea Mathematica, vol. 52, no. 2, pp. 127-155, 2001.

[17] H. Corrada, K. Leeb, B. Klein, R. Klein, S. Iyengarc, and G. Wahbad, "Examining the relative influence of familial, genetic, and environmental covariate information in flexible risk models," Proceedings of the National Academy of Sciences of the United States of America, vol. 106, no. 20, pp. 8128-8133, 2009.

[18] C. Arteaga and I. Marrero, "Universal approximation by radial basis function networks of Delsarte translates," Neural Networks, vol. 46, pp. 299-305, 2013.

[19] C. Arteaga and I. Marrero, "Approximation in weighted $p$-mean by RBF networks of Delsarte translates," Submitted Preprint, 2013.

[20] C. Arteaga and I. Marrero, "Wiener's tauberian theorems for the Fourier-Bessel transformation and uniform approximation by RBF networks of Delsarte translates," Submitted Preprint, 2013.
[21] J. Levesley and W. Light, "Direct form seminorms arising in the theory of interpolation by translates of a basis function," Advances in Computational Mathematics, vol. 11, no. 2-3, pp. 161$182,1999$.

[22] C. Arteaga and I. Marrero, "Direct form seminorms arising in the theory of interpolation by Hankel translates of a basis function," Advances in Computational Mathematics, 2013.

[23] J. J. Betancor and I. Marrero, "The Hankel convolution and the Zemanian spaces $\beta_{\mu}$ and $\beta_{\mu}^{\prime \prime}$ " Mathematische Nachrichten, vol. 160, pp. 277-298, 1993.

[24] A. H. Zemanian, Generalized Integral Transformations, Interscience Publishers, 1968.

[25] A. Erdélyi, W. Magnus, F. Oberhettinger, and F. Tricomi, Tables of Integral Transforms, McGraw-Hill, 1954.

[26] M. Abramowitz and I. A. Stegun, Handbook of Mathematical Functions with Formulas, Graphs, and Mathematical Tables, vol. 55 of Applied Mathematics Series, 9th printing, National Bureau of Standards, 1964. 


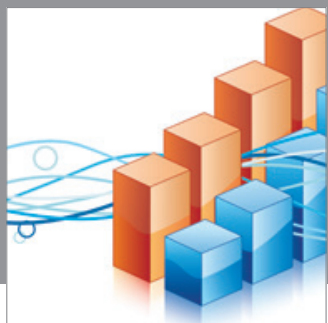

Advances in

Operations Research

mansans

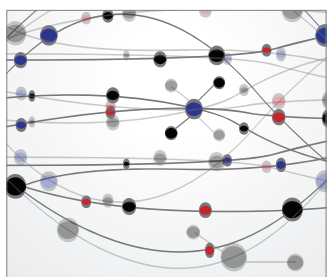

The Scientific World Journal
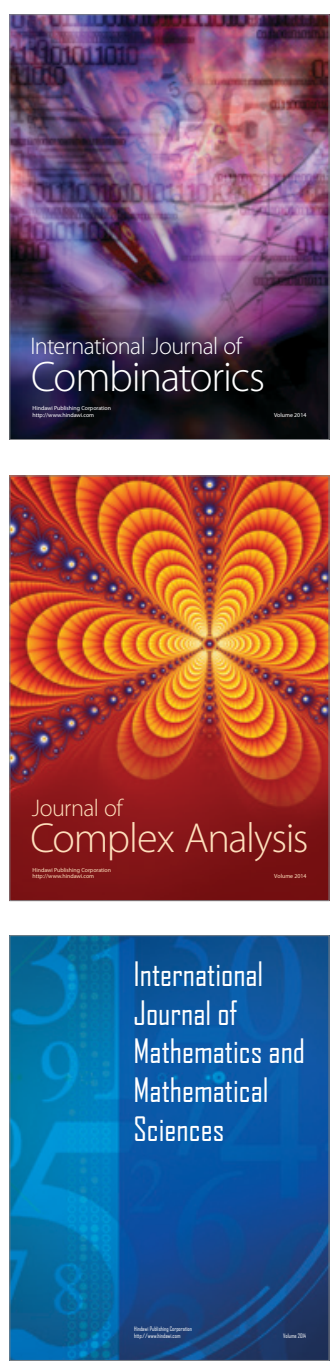
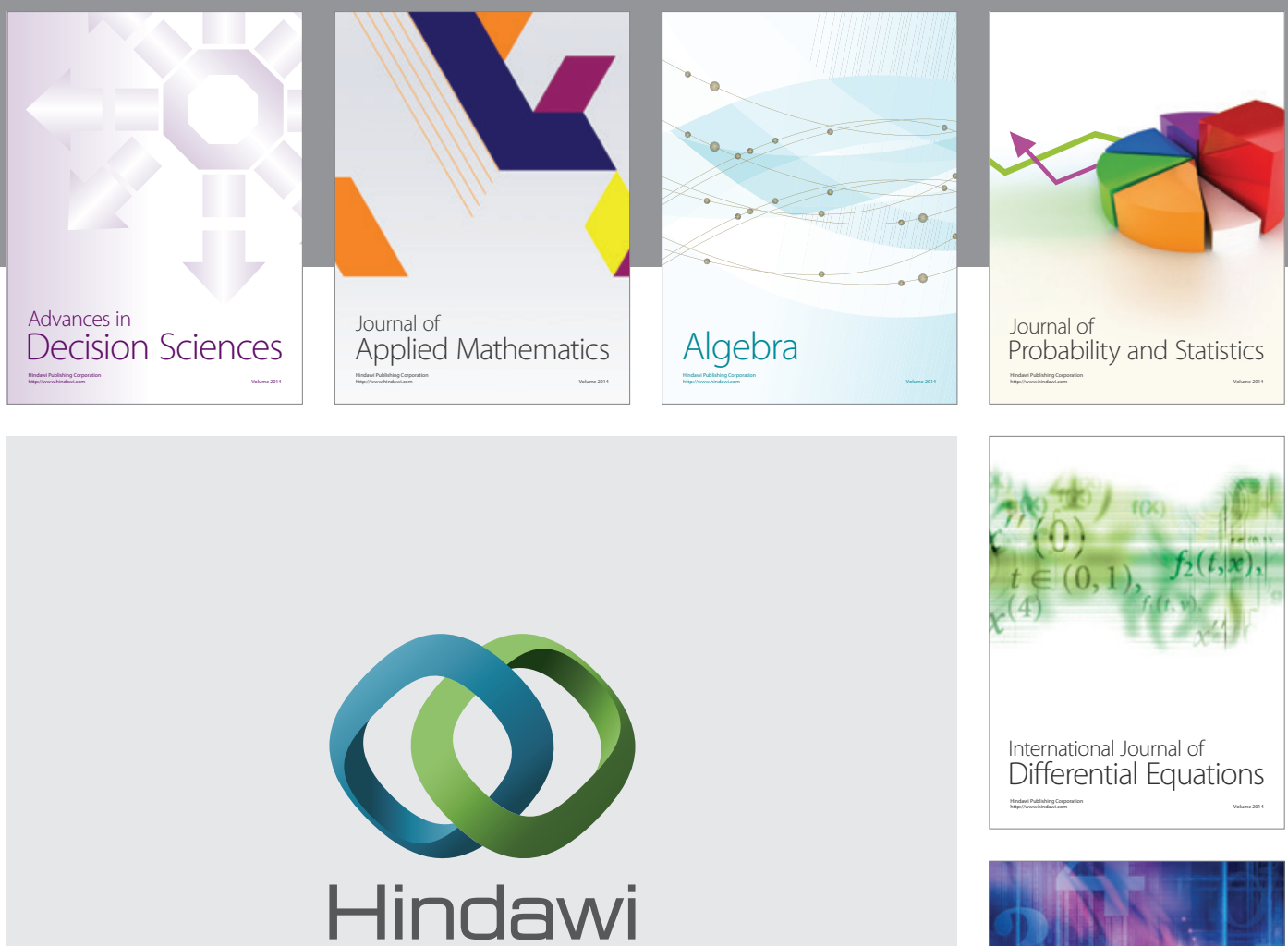

Submit your manuscripts at http://www.hindawi.com
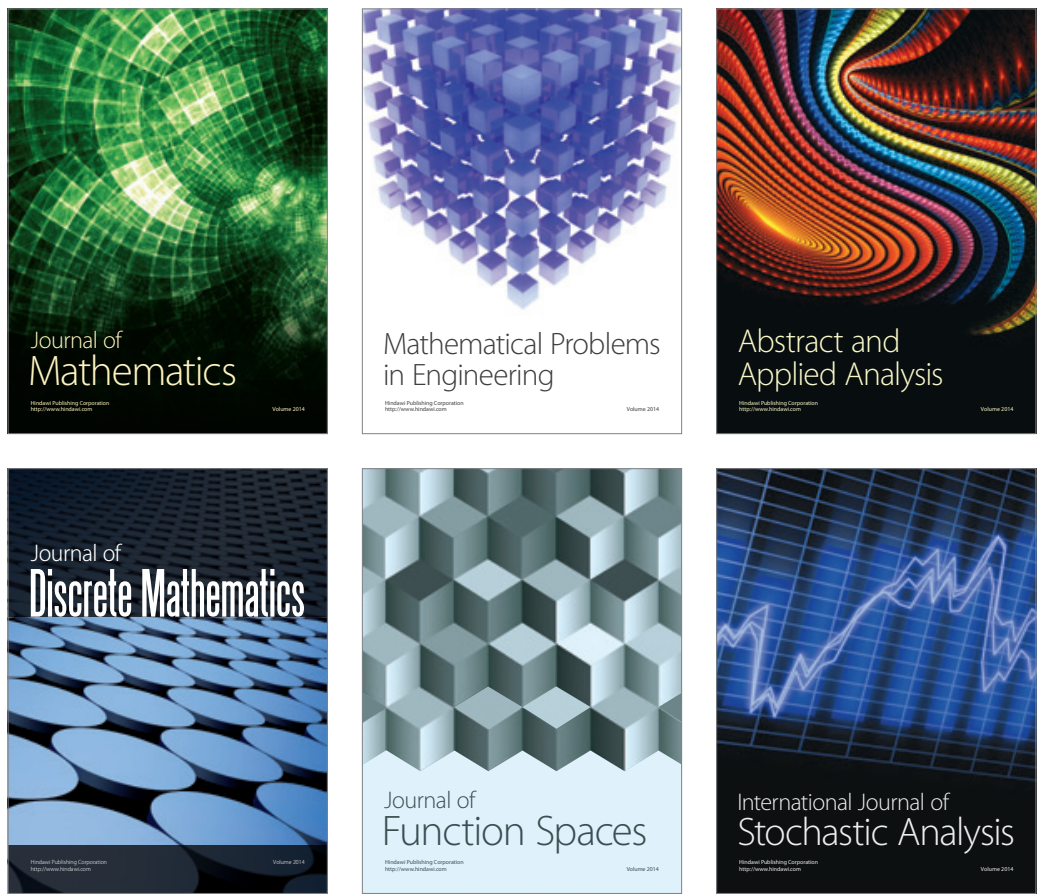

Journal of

Function Spaces

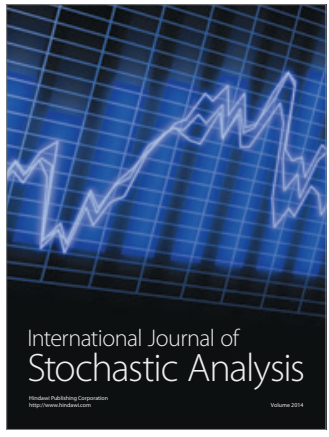

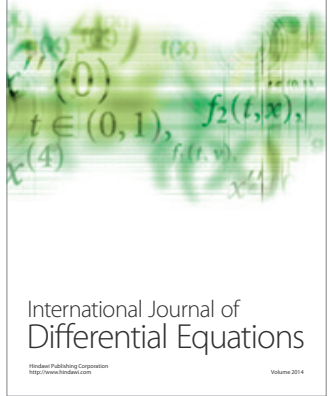
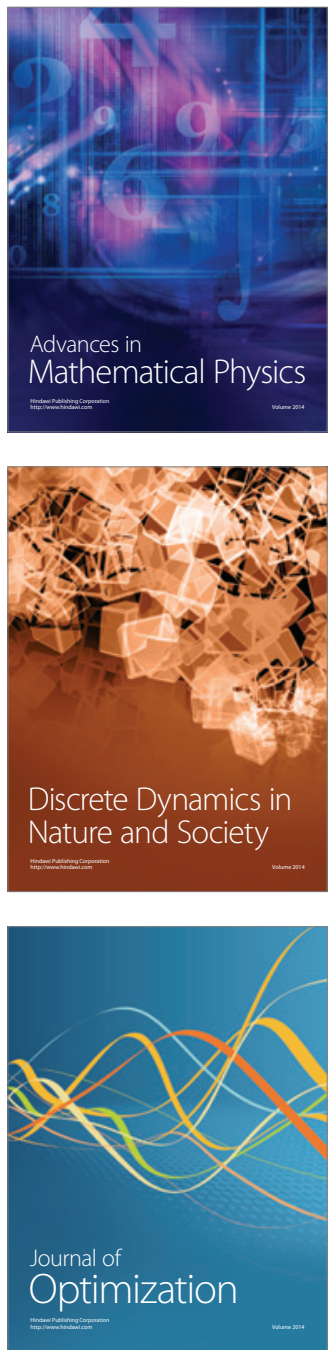